\title{
SOBERANÍA DEL CONSUMIDOR Y LIBERTAD DE ELECCIÓN EN PAÍSES EN DESARROLLO*
}

Gonzalo Ruiz Diaza

*DOI: https://doi.org/10.18601/01245996.v20n38.04. Este artículo es parte de la labor de investigación del autor como profesor asociado de la Pontificia Universidad Católica del Perú. Recepción: 25-05-2016, modificación final: 18-01-2018, aceptación: 19-02-2018. Sugerencia de citación: Ruiz D., G. (2018). Soberanía del consumidor y libertad de elección en países en desarrollo. Revista de Economía Institucional, 20(38), 71-95.

a Doctor en Economía. Director de la maestría en Regulación de Servicios Públicos de la Pontificia Universidad Católica del Perú, Lima, Perú, [gruiz@pucp.edu.pe]. 


\section{Soberanía del consumidor y libertad de elección en países en desarrollo}

Resumen. A partir de las nociones de libertad de proceso y de oportunidad de Amartya Sen, este artículo expone las condiciones necesarias para que el funcionamiento de los mercados tenga resultados compatibles con el concepto de la soberanía del consumidor formulado por Hutt. Estas condiciones están vinculadas al desarrollo de capacidades básicas de los consumidores, la promoción de mercados con opciones plurales y el acceso a mecanismos de justicia de consumo. Examina, además, el papel del Estado en el cumplimiento de esas condiciones en países en desarrollo.

Palabras clave: soberanía del consumidor, protección al consumidor, libertad de elección, regulación, libre competencia; JEL: D18, L49

\section{Consumer sovereignty and freedom of choice in developing countries}

Abstract. Based on Amartya Sen's notions of freedom of process and opportunity, this article explain the necessary conditions for the operation of markets to have results compatible with the concept of consumer sovereignty formulated by Hutt. These conditions are linked to the development of the basic capacities of consumers, the promotion of markets with plural options and the access to consumer justice mechanisms. It also examines the role of the State in meeting these conditions in developing countries.

Keywords: consumer sovereignty, consumer protection, freedom of choice, regulation, free competition; JEL: D18, L49

\section{Soberania do consumidor e liberdade de eleição em países em desenvolvimento}

Resumo. A partir das noções de liberdade de processo e de oportunidade de Amartya Sen, este artigo expõe as condições necessárias para que o funcionamento dos mercados tenha resultados compatíveis com o conceito de soberania do consumidor formulado por Hutt. Estas condições estão vinculadas ao desenvolvimento de capacidades básicas dos consumidores, a promoção de mercados com opções plurais e o acesso a mecanismos de justiça de consumo. Examina também o papel do Estado no cumprimento dessas condições em países em desenvolvimento.

Palavras-chaves: soberania do consumidor, proteção do consumidor, liberdade de escolha, regulação, livre concorrência; JEL: D18, L49 
Z1 concepto de soberanía del consumidor ha sido empleado con Cfrecuencia para hacer referencia al rol determinante que juegan las preferencias de los consumidores en la asignación de los recursos de una economía. Originalmente se le atribuye a William Hutt (1940a); sin embargo, sus antecedentes se remontan a Adam Smith (1776, p. 512), quien afirmaba que "el consumo es el único fin y propósito de toda la producción y [que] el bienestar del productor debería ser considerado solo en la medida en que sea necesario para atender el del consumidor". El concepto también ha sido empleado por diversos autores, como Von Mises (1949, p. 271), a partir de la analogía democracia-mercado, para afirmar que, al igual que en el caso de un elector que escoge a sus gobernantes en un proceso electoral, el consumidor a partir de sus decisiones de consumo define qué empresa $o$ qué servicio prevalecerá en el mercado.

La idea central detrás de la noción de soberanía del consumidor planteada por Hutt es que la producción es un medio para alcanzar un fin: la satisfacción de necesidades de los consumidores. Debido a que existen múltiples necesidades que atender y recursos productivos limitados, según Hutt existe una 'escasez natural', la misma que es 'administrada' por el mecanismo competitivo del mercado. En tal sentido, la actividad de producción se encuentra subordinada a la disciplina de mercado dictada por la competencia y las preferencias de los consumidores, que son los que en última instancia definen la participación y estructura de la oferta de bienes y servicios. Esta noción de 'escasez natural' se contrapone al concepto de 'escasez artificial', la cual estaría generada por las barreras o distorsiones que afectan la competencia o el mecanismo de precios en el mercado. Entre estas, Hutt (1940a, p. 260) menciona aquellas intervenciones del Estado o de las asociaciones de productores que pueden afectar el libre flujo de bienes y servicios en el mercado.

E1 concepto de soberanía de consumidor, en los términos de Hutt, se sustenta en el ejercicio de la libertad de elección por parte de este último. Es en el ejercicio de dicha libertad que el consumidor, con sus decisiones en el mercado, determina de qué modo se asignan los recursos productivos. Es sin embargo importante comprender claramente en qué consiste dicha libertad y qué medidas de política debe adoptar -o dejar de adoptar- el Estado a fin de asegurar el ejercicio de la libertad de elección por parte de los consumidores. En este aspecto, como se analizará, resulta pertinente revisar el concepto de libertad a partir de las dos dimensiones identificadas por Sen (1993): la libertad respecto del proceso de adopción de decisiones (libertad de proceso) 
y la libertad en términos de factibilidad del logro de oportunidades (libertad de oportunidad).

El presente artículo discute las implicancias del debate en torno a la relación entre soberanía del consumidor y libertad de elección, con especial énfasis en las condiciones que enfrentan los países en desarro1lo. Se postula que cierta literatura, en particular la proveniente de la tradición libertaria, ha puesto un énfasis excesivo en la relación entre la libertad de elección y la implementación de políticas de desregulación de mercados o laissez faire. Como se verá, dicha asociación resulta parcial e incompleta y es consecuencia de la especial importancia que dicha literatura atribuye al concepto de libertad en su faceta de proceso antes que en la de oportunidad. Así, a partir de una discusión sobre la noción de libertad, se plantea que en el caso de países en desarrollo la mencionada asociación entre soberanía del consumidor y políticas de laissez faire requiere ser revisada y complementada. En particular, a fin de dotar a la noción de soberanía del consumidor de un contenido consistente con el planteamiento original de Hutt, se proponen tres condiciones básicas que serían necesarias para poder asociar la soberanía del consumidor con una plena y efectiva libertad de elección de los consumidores. En primer término, se plantea que es necesario garantizar el acceso a un conjunto de bienes y servicios públicos destinados a permitir el desarrollo de capacidades básicas por parte de los ciudadanos. En segundo lugar, se señala la necesidad de que exista una oferta cuya estructura o composición asegure una pluralidad de opciones a disposición de los consumidores en los mercados. En tercer y último término, se afirma que es necesario que los usuarios tengan acceso efectivo a mecanismos legales de defensa de sus derechos a la información y a la idoneidad de los bienes o servicios recibidos.

En la primera sección se contrastan los conceptos de libertad de proceso y libertad de oportunidad y en la segunda se relacionan con la necesidad de asegurar el desarrollo de capacidades básicas entre los consumidores. En la tercera se aborda la problemática de las estructuras de mercado y de la manera en que la soberanía del consumidor está sujeta a la disponibilidad de una pluralidad de opciones. En la cuarta se fundamenta la necesidad de asegurar el acceso de los consumidores a sistemas de justicia de consumo. Por último, se presenta una síntesis del conjunto de condiciones necesarias para que la libertad de elección de los consumidores de países en desarrollo se pueda traducir en una efectiva soberanía en el mercado. 


\section{LIBERTAD DE PROCESO Y LIBERTAD DE OPORTUNIDAD}

El análisis de los factores que hacen que el ejercicio de la libertad de elección de los consumidores se traduzca en un estado en el cual los patrones de producción y el desempeño de la economía reflejen sus preferencias requiere de manera previa abordar una definición clara del concepto de libertad. Sen (1993) distingue dos facetas importantes del concepto de libertad. Una primera se relaciona con la autonomía y no interferencia de terceros en el proceso de decisión individual. La autonomía guarda relación con la capacidad de las personas de adoptar decisiones por sí mismas. La no interferencia, por su parte, es una noción que hace referencia a la no intrusión o invasión por parte de terceros (incluido el Estado) en el ámbito de las decisiones individuales.

Esta faceta de la libertad ha sido enfatizada por la corriente libertaria de pensamiento, la misma que pone de relieve la autonomía de las personas en su proceso de toma de decisiones y acciones, así como su derecho a que terceros no interfieran en dicho proceso. Esta noción es expresada claramente en la definición de libertad planteada por Hayek (1960), como un estado en el cual las personas no están sujetas a coerción alguna o a la dependencia arbitraria de la voluntad de otro u otros.

Una noción similar de libertad es planteada por otros autores de la escuela austriaca como Nozick $(1974)^{1}$. En el caso específico del concepto de soberanía del consumidor, diversos autores (p. ej., Waldfogel, 2005) han destacado el valor intrínseco que otorgan los consumidores a la autonomía en la adopción de sus decisiones de consumo. Ha sido esta acepción del concepto de libertad la empleada principalmente por la corriente de pensamiento libertaria, al asociar la soberanía del consumidor con doctrinas de laissez faire o de no intervención del Estado en las decisiones que adoptan los consumidores en el mercado.

De otro lado, la noción de libertad como oportunidad enfatiza la necesidad de considerar la posibilidad real de que las decisiones de las personas se puedan traducir en determinados resultados. Esta noción concibe la libertad en términos instrumentales, como la capacidad efectiva de alcanzar aquella opción de mayor preferencia o bienestar

${ }^{1}$ Nozick (1974, p. 23), citando al filósofo Locke, señala: “Los límites del derecho natural exigen que 'nadie deba dañar a otro en su vida, salud, libertad o posesión' (sec. 6). Algunas personas transgreden estos límites 'invadiendo el derecho de otros [...] y haciéndose daño unas a otras' y, en respuesta, la gente puede defenderse, o defender a otro, contra los que violan derechos (cap. III)". 
para el consumidor, dentro de un conjunto de alternativas que este tenga a su disposición. En este caso no resulta tan relevante el proceso de decisión en sí mismo, en la medida que no se traduzca en la posibilidad de acceder a opciones que maximicen el bienestar o la satisfacción del consumidor. Para Sen (1993) esta faceta de la libertad no puede ser soslayada frente a la de proceso, pues ello equivaldría a privilegiar la supuesta autonomía de las personas en su proceso de decisión frente a su posibilidad (oportunidad) efectiva de acceder a determinadas funcionalidades mínimas:

La línea de razonamiento libertaria en realidad no puede ser independiente de los resultados. El tema adquiere particular importancia cuando las consecuencias derivadas del ejercicio de los derechos libertarios y la asignación de mercado son especialmente pobres en términos de bienestar individual, o en términos de libertad individual juzgada desde la perspectiva de la 'oportunidad de lograr'. De hecho, es posible que incluso hambrunas a gran escala se produzcan sin violar los derechos libertarios de nadie y sin apartarse del funcionamiento de un mecanismo de libre mercado. La alegada independencia de resultados de los derechos libertarios está sujeta por tanto a cuestionamientos severos en términos de aceptabilidad ética. (Sen, 1993, p. 526)

Sen (1993) considera éticamente cuestionable sostener que una persona que no puede satisfacer ciertas necesidades básicas sea libre de decidir. En esto se aparta drásticamente de la noción libertaria según la cual la libertad tiene un valor en sí mismo, independiente de los resultados ${ }^{2}$. Esto hace que Sen, como otros autores, otorgue especial importancia a la posibilidad de realización de las capacidades humanas como componente esencial de la libertad.

Un arreglo social en el que la libertad en sus dos facetas (de proceso y de oportunidad) se cumpla para todos sus ciudadanos implica la promoción de políticas que sopesen los derechos a la no interferencia en las decisiones privadas y la posibilidad real de que estos tengan

${ }^{2}$ Hayek (1944, pp. 47-48), por el contrario, identifica la faceta de oportunidad, antes que con el concepto de libertad, con demandas para la redistribución de la riqueza: "Para hacer que este argumento suene plausible, la palabra 'libertad' ha sido sometida a un cambio sutil en el significado. La palabra que anteriormente significaba libertad de la coacción, del poder arbitrario de otros hombres, ahora se ha transformado para significar libertad de la necesidad, liberación de la compulsión de las circunstancias que inevitablemente limitan el rango de elección de todos nosotros. La libertad en este sentido es, por supuesto, simplemente otro nombre para el poder o la riqueza. Las demandas por esta nueva libertad son, pues, solo otro nombre para denominar a las viejas demandas de redistribución de la riqueza" (trad. propia). 
una capacidad mínima de alcanzar determinados resultados ${ }^{3}$. Uno de los esfuerzos más importantes desplegados desde el liberalismo por desarrollar el concepto de igualdad de oportunidades proviene de Rawls, quien en sus contribuciones Una teoría de la justicia (1971) y El liberalismo politico (1993) propugna como primer principio de justicia que todos los miembros de la sociedad tengan acceso a un conjunto de bienes primarios, entre los cuales destacan la libertad política (de votar y de postularse a cargos de elección), la libertad de expresión y de asociación, la libertad de conciencia y la libertad de pensamiento, así como la libertad de poseer propiedad personal. Rawls plantea que todos los miembros de la sociedad tengan un acceso mínimo a estos bienes primarios $^{4}$ para asegurar el ejercicio de su libertad. Sen (2005) critica esta noción señalando que otorga una importancia desproporcionada al acceso a bienes (al punto que la denomina el 'fetichismo' del consumo de bienes), en lugar de privilegiar las funcionalidades o potencialidades que estos otorgan a las personas. En este sentido, para Sen no importa tanto el conjunto de bienes y servicios a los que las personas tienen acceso sino lo que estas pueden hacer con ellos. Antes que los bienes y servicios que las personas tienen a su disposición, para Sen lo que importa son las funcionalidades que estos les otorgan: sus capacidades. Así, por ejemplo, una persona que goce a plenitud de todas sus facultades físicas y una persona que tenga algún tipo de discapacidad pueden tener acceso igualitario a un conjunto de bienes y servicios, pero las capacidades que se deriven del acceso a dichos bienes para ambas serán diferentes. De allí que igualar oportunidades, bajo ciertas condiciones, pueda implicar posibilitar el acceso a ciertos individuos a un conjunto mayor o diferente de bienes y servicios que a otros.

La idea de establecer condiciones mínimas que permitan al consumidor elegir libremente no es contraria al concepto original de soberanía del consumidor planteado por Hutt, tal como lo reconoce Persky (1993, p. 190).

Por el contrario, para Hutt, un requisito para que el funcionamiento del mercado se traduzca en una efectiva soberanía del consumidor consiste en que deben darse condiciones que aseguren la participación de los ciudadanos bajo condiciones mínimas de igualdad de oportu-

${ }^{3}$ Para una revisión del debate en torno al concepto de igualdad de resultados e igualdad de oportunidades ver Roemer y Tranoy (2013).

${ }^{4}$ A Rawls se le atribuye la función de bienestar denominada "maximin" como un mecanismo de medición de funciones de bienestar; sin embargo, el autor concibió dicho concepto para garantizar una adecuada distribución de los bienes primarios que asegure igualdad de oportunidades entre los miembros de una sociedad. 
nidad, de manera similar al modo en que ocurre en una democracia. En diversos pasajes de The economists and the public Hutt se muestra partidario de la adopción de medidas que desde el Estado promuevan igualdad de oportunidades, como el establecimiento de un esquema progresivo de impuestos o la existencia de sistemas de crédito público a la educación. Más aún, en ciertos pasajes, Hutt (1940, p. 313) llega a afirmar que la igualdad de oportunidades constituye un fin en sí mismo y que afirmar lo contrario sería inconcebible con el funcionamiento de un sistema democrático.

Para Hutt, la igualdad de oportunidades no solo es un medio para que el desempeño del mercado y la oferta reflejen genuinamente la voluntad de los consumidores, sino también para promover una sociedad más tolerante dotando de legitimidad al desempeño resultante de la dinámica del mercado.

En The economists and the public Hutt se aparta de la noción libertaria de la existencia de un 'estado natural' de libertad absoluta (no interferencia), pues, al igual que Von Mises (1949) ${ }^{5}$, admite que en la medida que el hombre interactúe en el contexto de una sociedad, inevitablemente las decisiones de unos afectarán las posibilidades de decisión de otros. No obstante, resalta que dichas restricciones a la libertad no deben ser arbitrarias, por el contrario, deben ser imparciales e impersonales ${ }^{6}$. El carácter imparcial e impersonal de las

5 Von Mises (1960, p. 280) usa una definición similar a la de Nozick, aunque discrepa con respecto a que dicha libertad forme parte de un estado natural, tal como lo refiere dicho autor: "La libertad siempre se refiere a las relaciones interhumanas. Un hombre es libre en la medida en que puede vivir y progresar sin estar a merced de decisiones arbitrarias de otras personas. En el marco de la sociedad, todos dependen de sus conciudadanos. E1 hombre social no puede independizarse sin abandonar todas las ventajas de la cooperación social. El individuo autosuficiente es independiente, pero no es libre. Está a merced de todos los que son más fuertes que él. El hombre más fuerte tiene el poder de matarlo con impunidad. Por lo tanto, es absurdo despotricar sobre una supuesta libertad 'natural' e 'innata' de la que se supone que las personas disfrutaron en las edades anteriores al surgimiento de los vínculos sociales. El hombre no fue creado libre; la libertad que pueda tener le ha sido dada por la sociedad. Solo las condiciones sociales pueden dotar a un hombre de una órbita dentro de cuyos límites puede alcanzar la libertad".

${ }^{6}$ Hutt (1940, pp. 252-253) señala: "La experiencia nos dice que los individuos (incluso aquellos que tienen una mentalidad crítica) no resienten las restricciones ni las reconocen como tales cuando se las considera inevitables, imparciales o, lo que es casi lo mismo, cuando se las considera impersonales. De ahí que los conceptos de 'libertad' y justicia estén estrechamente relacionados entre sí. No se considera que las restricciones que consideramos justas limiten nuestra libertad".

Revista de Economía Institucional, vol. 20, N. ${ }^{\circ}$ 38, Primer semestre/20i 8, pp. 7i-95 
restricciones a las libertades, las cuales se concretan por medio de un mercado verdaderamente competitivo, según Hutt, promueven una mayor aceptación y tolerancia en la sociedad, siendo el único mecanismo legítimo de coerción al que deben estar sujetos los ciudadanos y los productores de bienes y servicios.

\section{LIBERTAD DE ELECCIÓN Y DESARROLLO DE CAPACIDADES}

Teniendo en cuenta que, de acuerdo al espíritu de la propuesta de Hutt, el logro de una auténtica soberanía del consumidor requiere necesariamente de ciertas condiciones básicas que aseguren la libertad de los ciudadanos en sus dos facetas (de proceso y de oportunidad), ¿existe algún rol para el Estado en asegurar dichas libertades?, ¿qué rol le compete al Estado a fin de asegurar a sus ciudadanos igualdad de oportunidades? En la presente sección sostenemos que es indispensable que, a efectos de dotar al consumidor de herramientas que le permitan ejercer su libertad de elección, este pueda acceder a un conjunto de mecanismos que posibiliten el desarrollo de sus capacidades.

Como se mencionó en la sección anterior, la faceta de oportunidad del concepto de libertad está estrechamente vinculada con la posibilidad de las personas de acceder a determinadas funcionalidades o capacidades. Anand y Ravallion (1993, p. 135) resaltan que en el caso de países en desarrollo, las aproximaciones al problema se han centrado en la medición de la satisfacción de ciertas necesidades consideradas básicas, como condición que garantiza el acceso a ciertas 'funcionalidades elementales'. Así, por ejemplo, el "enfoque de capacidades", según dichos autores, a fin de medir niveles de bienestar en países en desarrollo, lejos de centrarse en logros efectivos alcanzados por la población, se enfoca en variables tales como esperanza de vida, niveles de morbilidad, tasas de mortalidad, analfabetismo, desnutrición y acceso a libertades cívicas.

El enfoque de capacidades contrasta sustancialmente con el formulado por Rawls, quien plantea como condición primigenia que los ciudadanos accedan a un conjunto de bienes primarios. Lo mismo ocurre en el caso del enfoque de "necesidades básicas insatisfechas" (Feres y Mancero, 2001), en la medida que este hace referencia a funcionalidades antes que a bienes. Tal como destacan Anand y Ravallion (1993), los nuevos enfoques de desarrollo humano (que llevan implícita la noción de libertad, con especial énfasis en su faceta de oportunidad) vinculan dicho desarrollo con el acceso a funcionalidades que dependen en gran medida de la provisión de servicios públicos. 
E1 Índice de Desarrollo Humano (IDH), según estos autores, recoge el enfoque de capacidades de Sen y refleja la noción de que mayores niveles de IDH están vinculados con un mayor acceso a servicios públicos. El anexo muestra el nivel de correlación existente entre el $\mathrm{IDH}^{7}$ e indicadores de acceso a servicios públicos tales como agua y saneamiento (Unicef, 2015), tasa de analfabetismo (Unesco, 2015), acceso a energía eléctrica (Banco Mundial,2012) y tasa de incidencia de TBC (OMS, 2014) para una muestra de países de Latinoamérica y el Caribe y países desarrollados.

En todos los casos, los gráficos permiten apreciar la existencia de una correlación positiva entre el IDH y el acceso a servicios públicos, como agua y saneamiento, educación, salud y acceso a energía. Asimismo, es posible apreciar que en todos los casos los países de Latinoamérica y el Caribe se encuentran rezagados respecto de los países desarrollados incluidos en la muestra. Esta correlación no implica necesariamente causalidad, pero evidencia que existe una clara asociación entre el desarrollo de capacidades básicas y el desarrollo humano.

La existencia de una asociación entre desarrollo de capacidades básicas y desarrollo humano es soportada desde distintas disciplinas. Por ejemplo, desde la psicología, Maslow (1943), creador de la teoría de jerarquización de necesidades, distingue cinco niveles de necesidades: fisiológicas, de seguridad, de afiliación, de reconocimiento y de autorrealización. El acceso a servicios básicos en cantidad y con calidad suficientes constituye un medio para lograr la satisfacción de necesidades básicas, en especial las fisiológicas y de seguridad ${ }^{8}$.

Si bien el aporte de Maslow proviene de la psicología y tiene una finalidad distinta, ha sido utilizado por diversos autores para discutir los conceptos de libertad y su relación con la medición del bienestar (Islam y Clarke, 2001). En términos de Maslow (1943), la no satisfacción de ciertas necesidades básicas puede limitar de manera sustancial el logro de otros objetivos superiores de las personas. En este sentido, la no satisfacción de necesidades básicas puede inhibir el interés y el esfuerzo en la consecución de otras necesidades menos urgentes:

${ }^{7}$ Los datos del IDH provienen del PNUD y corresponden al año 2014.

${ }^{8}$ Maslow distingue cinco niveles de necesidades: fisiológicas (respiración, alimentación, descanso, sexo, homeostasis), seguridad (seguridad física, de empleo, de recursos, moral, familiar, de salud, de propiedad privada), afiliación (amistad, afecto, intimidad sexual), reconocimiento (autorreconocimiento, confianza, respeto y éxito) y autorrealización (moralidad, creatividad, espontaneidad, falta de prejuicios, aceptación de hechos, resolución de problemas).

Revista de Economía Institucional, vol. 20, n. ${ }^{\circ}$ 38, Primer semestre/2oi 8, pp. 7 i-95 
Si todas las necesidades están insatisfechas, y el organismo está dominado por las necesidades fisiológicas, todas las demás necesidades pueden simplemente desaparecer o dejarse de lado. Entonces es justo caracterizar a todo el organismo diciendo simplemente que está hambriento, porque el hambre casi anula por completo la conciencia. Todas las capacidades se ponen al servicio de la satisfacción del hambre, y la organización de estas capacidades está casi totalmente determinada por el único propósito de satisfacer el hambre. Los sentidos receptores y emisores, la inteligencia, la memoria, los hábitos, todos ahora pueden definirse simplemente como herramientas que gratifican el hambre. Las capacidades que no son útiles para este propósito permanecen inactivas, o son empujadas al fondo. El deseo de escribir poesía, el deseo de adquirir un automóvil, el interés en la historia de Estados Unidos, el deseo de un nuevo par de zapatos son, en el caso extremo, olvidados o de importancia secundaria. Para el hombre que está extremada y peligrosamente hambriento, no existen otros intereses sino la comida. Sueña con la comida, recuerda la comida, piensa en la comida, solo se da cuenta de la comida, solo percibe comida y solo quiere comida. Los determinantes más sutiles que ordinariamente se fusionan con los impulsos fisiológicos para organizar incluso la alimentación, la bebida o el comportamiento sexual, ahora pueden estar tan abrumados que nos permiten hablar en este momento (pero solo en este momento) del impulso y apetito de hambre puros, con el único objetivo incondicional de alivio. (Maslow, 1943, p. 5)

En este sentido, a fin de permitir que los consumidores puedan ejercer su libertad de elección, las sociedades deben posibilitar el acceso a los bienes, servicios y facilidades que les permitan satisfacer a plenitud dichas necesidades básicas. Con relación a las necesidades fisiológicas, resulta indispensable que las personas gocen de una adecuada nutrición y alimentación durante todas sus etapas de vida, de manera que no se vea comprometido su desarrollo físico y síquico. Del mismo modo, aunque no es mencionado de manera expresa por Maslow ${ }^{9}$, debe proveerse al ciudadano de niveles de educación básica a fin de que este desarrolle una capacidad cognitiva que le permita desenvolverse adecuadamente en sociedad. En cuanto a los mecanismos de protección, estos resultan indispensables para que el ciudadano

${ }^{9}$ La teoría motivacional de Maslow no ha estado exenta de debate. Algunos autores como Wahba y Bridell (1976) han revisado diversos trabajos en los que no se ha encontrado evidencia concluyente en favor de dicha teoría. Contribuciones más recientes (Kenrick et al., 2010) han reformulado dicha teoría sosteniendo que el carácter de la jerarquización de necesidades planteada por Maslow no implica que una vez satisfecho un nivel de necesidades este desaparece, dando paso al siguiente nivel, sino que este último se superpone al primero. Es decir, necesidades como las fisiológicas o de seguridad no desaparecen sino que se mantienen, y a ellas se superponen otras como las de afiliación, reconocimiento y autorrealización. 
cuente con los medios de protección de su propiedad privada (entre otros, sus bienes de consumo o su ahorro) frente al potencial robo, expropiación, así como cualquier atentado contra su vida o su salud. Estos dos niveles de necesidades, en nuestro concepto, constituirían el umbral mínimo por encima del cual un consumidor podría ejercer su libertad de elección en el mercado. Por debajo de estos umbrales, las capacidades y libertades del consumidor se verían reducidas o limitadas, enfocándose el esfuerzo de este en la satisfacción de sus necesidades básicas.

El reconocimiento de que existen un conjunto de necesidades básicas, sin las cuales el desarrollo de las personas puede verse limitado, ha sido recogido en diversos ordenamientos políticos y constitucionales, los cuales han establecido como una de las funciones del Estado el garantizar condiciones de igualdad de oportunidades para sus ciudadanos. Así, el acceso a la salud, la seguridad, la educación, el agua potable y la vivienda, entre otros, ha sido establecido como una obligación del Estado en diversos ordenamientos jurídicos, como los europeos y los latinoamericanos, bajo el concepto de "servicio público" (Melián, 2005).

En efecto, siendo el desarrollo de capacidades humanas de los ciudadanos considerado una responsabilidad del Estado, en diversos países se han adoptado arreglos institucionales a partir de los cuales este asume directamente la provisión de ciertos servicios, o bien se contempla algún tipo de colaboración público-privada para asegurar dicha provisión. Es claro que, sea cual fuere dicho arreglo, la intervención del Estado a través del financiamiento, el otorgamiento en concesión de ciertos servicios o la provisión directa de dichos bienes en cantidad y con calidad suficientes resulta crucial, no solo para el desarrollo de las capacidades humanas de sus ciudadanos, sino para el pleno ejercicio de sus libertades de elección. Esta necesidad de participación del Estado se hace más clara y patente en aquellas ramas de producción o servicio en las que no existen empresas privadas dispuestas a ofrecer el bien o servicio, o en las que la rentabilidad privada resulta insuficiente para compensar el costo privado de oportunidad del capital.

Por ello es que en los países en desarrollo las políticas que promuevan el acceso universal a servicios públicos como electricidad, agua potable y saneamiento, comunicaciones, salud, educación y seguridad, constituyen el punto de partida de cualquier enfoque que busque poner a los ciudadanos en condiciones mínimas que les permitan el ejercicio pleno de sus capacidades. 
Es importante tener en cuenta que un enfoque que garantice el acceso universal a este conjunto de bienes y servicios básicos debe tener en cuenta la diversidad de necesidades y características de las personas. Resulta evidente, por ejemplo, que en el caso de personas con habilidades especiales o discapacidades se requerirá un conjunto de servicios diferentes al del resto de ciudadanos. Igualmente en lo que respecta a las distintas necesidades relacionadas con el enfoque de género. Lo esencial es que, teniendo en cuenta dicha diversidad, las personas puedan contar con medios que les permitan satisfacer sus necesidades básicas, y que ello a la vez posibilite el pleno desarrollo de sus capacidades.

Contrariamente a lo que la prédica libertaria preconiza, la intervención del Estado en el aseguramiento del acceso a estos bienes y servicios básicos, antes que restringir, puede potenciar las capacidades y libertades de elección en su faceta de oportunidad. En algunos casos, como ya se mencionó, dicha intervención toma la forma de políticas de acceso universal; en otros, la de provisión directa del bien o servicio por parte del Estado. En estos casos, no es el laissez faire o la no intervención lo que promueve la libertad de elección y la soberanía del consumidor, sino, por el contrario, una participación del Estado supliendo la falta de oferta privada de servicios básicos o asegurando la provisión de los bienes en cantidad y con calidad suficientes.

Cabe resaltar que el hecho de que existan cierto tipo de necesidades que resulta indispensable satisfacer no implica necesariamente que sea únicamente el Estado el que las deba suplir, ni que cualquier tipo de intervención en este ámbito esté justificada. Desde una perspectiva económica existen un conjunto de posibles fórmulas público-privadas que pueden resultar más costo-eficientes por implicar mayores beneficios netos para la sociedad. En este sentido, la existencia de necesidades básicas no exonera al Estado de hacer caso por caso un cuidadoso análisis costo-beneficio de sus intervenciones.

En síntesis, el conjunto de oportunidades de consumo para un ciudadano que ha tenido acceso a servicios de salud, educación y servicios públicos, entre otros, será sin duda mayor que el de aquel que no ha podido acceder a los mismos. Este abanico de posibilidades de consumo sería inalcanzable sin el acceso a esta 'canasta básica' de servicios, resultando ello un requisito esencial para el ejercicio de la libertad de elección y de la soberanía de los consumidores. En muchos casos, aunque no necesariamente siempre, dicho objetivo es alcanzable por medio de alguna forma de intervención del Estado, sea en el financiamiento o en la provisión de dichos bienes, antes que con políticas de laissez 
faire. En aquellos casos en que el Estado promueva la provisión privada de dichos bienes y la estructura de mercado, por razones económicas o estructurales, no posibilite la competencia, este debe asegurar por medio de la regulación que dichos servicios se presten en condiciones mínimas de calidad y precio que aseguren los objetivos de acceso y servicio universal discutidos en la presente sección.

\section{LIBERTAD DE ELECCIÓN Y PLURALIDAD DE OPCIONES}

E1 funcionamiento de una economía de mercado se basa en tres hipótesis descriptivas básicas de comportamiento: la competencia perfecta, la plena descentralización de las decisiones individuales y la propiedad privada.

Los cursos básicos de microeconomía demuestran que uno de los principales obstáculos al ejercicio de la soberanía del consumidor surge cuando el primero de los supuestos mencionados falla: en el extremo, cuando existe un monopolio. Asimismo, algunos autores de la escuela austriaca como von Mises (1949, p. 358) señalan expresamente que "los precios de monopolio constituyen una violación de la supremacía de los consumidores y de la democracia de mercado" 10 .

Es decir, en aquellos casos en los que la estructura del mercado sea monopólica, el consumidor deja de ser quien con sus preferencias determina qué se produce y en qué cantidad: deja de ser el soberano. Asimismo, su libertad de elección se verá limitada por la falta de opciones para elegir.

Desde una perspectiva normativa, el enfoque microeconómico tradicional se ha centrado en los efectos de esta estructura de mercado en términos de asignación de recursos, identificando como el principal problema asociado a esta estructura de mercado la pérdida de eficiencia sobre la asignación de recursos (Posner, 1974), antes que objetivos asociados a la protección de libertades de elección o del ejercicio de la soberanía del consumidor. También la literatura reconoce que en países en desarrollo los efectos asignativos de la ausencia de políticas consistentes de competencia y la presencia de estructuras de mercado concentradas pueden ser más severos ${ }^{11}$.

${ }^{10}$ La concepción de los economistas de la escuela austriaca no es uniforme respecto a la naturaleza del monopolio. Por ejemplo, Rothbard critica la noción de Von Mises y Hutt sobre el monopolio y la noción de soberanía del consumidor, y contrapone a esta última la noción de 'soberanía del individuo'; ver Gunning (2009).

11 Godfrey (2008) plantea un conjunto de hipótesis respecto a los canales de transmisión a través de los cuales la política de competencia promueve el crecimiento y reduce la pobreza en países en desarrollo. Filho (2015), me- 
No obstante, buena parte de las recomendaciones o prescripciones de política que se desprenden de esta literatura contribuyen también al logro de la promoción de la igualdad de oportunidades. Así, por ejemplo, las políticas antimonopolio implementadas en la mayoría de países con economías de mercado, si bien se orientan a prevenir o corregir prácticas empresariales consideradas anticompetitivas, contribuyen también al objetivo de que los consumidores puedan preservar su derecho a elegir entre una pluralidad de opciones. Sin perjuicio de lo anterior, en el contexto de las políticas antitrust ha existido un extenso debate respecto a que dichos objetivos deben sopesarse con otros vinculados con eficiencia productiva o innovación (Wandel, 2012). En muchos casos, el razonamiento de las agencias antimonopolio o de las cortes ha justificado conductas empresariales que privilegian el aprovechamiento de eficiencias productivas o dinámicas, pudiendo afectar en el corto plazo la existencia de una pluralidad de opciones en el mercado. Por ejemplo, ciertas prácticas de atadura de productos o servicios han sido justificadas ${ }^{12}$ por las eficiencias dinámicas que estas generan y que pudieran, al menos temporalmente, ocasionar una reducción en el conjunto de opciones que tiene el consumidor para elegir (Williamson, 1968).

Mención aparte merece el caso de aquellos mercados en los que factores tecnológicos o de escala únicamente permiten que exista un solo ofertante. Este es el caso de las llamadas facilidades esenciales (Cotter, 2010). En estos casos, la política antitrust, a través de medidas excepcionales, puede intervenir a fin de evitar que el poder de mercado del ofertante afecte negativamente la competencia y el funcionamiento del mercado. Igualmente en el caso de industrias con características de monopolio natural, en las que por razones estructurales o tecnológicas no es posible la competencia, se han desarrollado un conjunto de instrumentos de regulación económica con la finalidad de establecer mecanismos que impidan que el poder de mercado afecte el acceso de los consumidores a dichos servicios.

Existe relativo consenso en que uno de los obstáculos que impide el ejercicio de la soberanía del consumidor es la existencia de estructuras de mercado monopólicas. En estos casos, nuevamente apreciamos

diante un análisis histórico, explica de qué manera los países que han sido colonias han heredado estructuras monopólicas de mercado que, entre otras cosas, han perpetuado su situación de subdesarrollo.

${ }^{12}$ E1 uso de este tipo de razonamientos por las autoridades en el caso de Estados Unidos no ha estado exento de críticas, incluso del propio Hutt (1940a, pp. 360-361) cuando hace referencia al caso Standard Oil y al uso de la denominada Regla de la Razón. 
que las políticas de laissez faire o de no intervención pueden resultar contrarias al objetivo de garantizar la libertad de elección y la soberanía del consumidor. Políticas antitrust y en ocasiones de regulación en el caso de facilidades esenciales que promuevan la competencia, son necesarias para asegurar que el mercado brinde a los consumidores condiciones para el ejercicio de una efectiva libertad de elección. Como resalta Yamey (1988), el propio Hutt se muestra partidario de una activa política antitrust como un mecanismo que promueve las libertades de elección de los consumidores.

Sin embargo, en el objetivo de acceso por parte de las consumidores a una amplia pluralidad de opciones no solo debemos considerar las políticas antimonopolio o antitrust, sino el conjunto de políticas públicas que contribuyen a dicho fin, como la desregulación de mercados y/o la remoción de barreras a la entrada. El acceso por parte de los consumidores a una pluralidad de opciones no es posible solo a través de la política antitrust, requiriéndose un conjunto de herramientas de política económica que complementen la labor de las agencias de competencia. Puede ser que la existencia de un monopolio haya estado originada en una barrera a la entrada impuesta desde el propio Estado. En estos casos, lo conveniente sería remover el origen de la distorsión antes que sus consecuencias. De manera similar a lo mencionado en la sección anterior, el tipo de intervención del Estado debe basarse en un análisis riguroso costo-beneficio de cada caso.

\section{LIBERTAD DE ELECCIÓN Y PROTECCIÓN AL CONSUMIDOR}

Como ya se mencionó, existe consenso acerca de que la existencia de competencia efectiva fortalece la capacidad de elección de los consumidores ${ }^{13}$. Hutt creía que la mejor defensa del consumidor ante prácticas fraudulentas en su contra es una férrea promoción de la competencia. Incluso fue más allá y afirmó que las demandas para regular este tipo de prácticas suelen ser promovidas por grupos opuestos a la competencia ${ }^{14}$.

No obstante, existen al menos dos factores que impiden que la competencia por sí sola garantice un mercado totalmente transpa-

${ }^{13}$ Para un análisis de las áreas complementariedad y tensión entre la protección al consumidor y las políticas de competencia, ver Cseres (2005).

${ }^{14}$ Hutt (1940, p. 103): "Creemos que en los hechos, la instituciones promotoras de la competencia son la salvaguarda más efectiva contra la prevalencia del fraude". 
rente, con productos y servicios prestados de manera idónea ${ }^{15}$ : las asimetrías de información entre proveedores y consumidores en un grupo importante de mercados y las asimetrías en la negociación. Como se plantea a continuación, sin políticas públicas que doten a los consumidores de acceso a la justicia de consumo, previniendo o resolviendo aspectos vinculados con su derecho a la información y a recibir servicios idóneos, difícilmente se estará en capacidad de asegurar que la estructura de producción responda fehacientemente a las preferencias de los consumidores.

En el primer caso, Akerlof (1970) demuestra cómo en un mercado competitivo pero en el que existe asimetría de información, en particular, problemas de selección adversa, los consumidores fallan en distinguir productos de "buena" y "mala" calidad. Más allá de los efectos asignativos que ocasiona esta situación (enfatizados por dicho autor), el ejemplo es útil para mostrar cómo en contextos de asimetría de información no basta la libre competencia para que los consumidores puedan adoptar decisiones informadas y acceder a los bienes o servicios que realmente desean consumir. La falta de información sobre atributos relevantes de los bienes y servicios se puede constituir en un obstáculo que impide el pleno ejercicio de la libertad (o capacidad) de elección, entendiendo esta última como la posibilidad de adoptar decisiones de consumo mínimamente informadas. Los efectos de la asimetría de información sobre dicha capacidad de elección son más severos en cierto tipo de bienes que en otros. Así, por ejemplo, Nelson $(1970 ; 1974)$ distingue entre los bienes búsqueda (aquellos cuyos atributos relevantes son perceptibles para el consumidor de manera inmediata) y los bienes experiencia (aquellos que requieren que el consumidor atraviese una primera experiencia de consumo). Darby y Karny (1973) añaden más tarde una tercera categoría denominada bienes "creencia" (credence) o "confianza", en los cuales aun en el caso

15 En ciertos pasajes de The economist and the public, Hutt precisa que es importante introducir algunas restricciones a la libertad de elección de los consumidores en defensa de sus propios intereses, bien sea debido a su posible falta de capacidad para discriminar bienes diversos o para elegir de manera informada aquellos bienes cuyo consumo puede afectar derechos de otros consumidores: "podemos concluir que en ciertas situaciones, el ejercicio del libre albedrío de una persona adulta, aunque en ningún sentido implique una frustración de la voluntad de otros, puede ser legítimamente restringido. La restricción es en su propio interés" (trad. propia). No obstante, en otros pasajes (ibíd., p. 278), Hutt es partidario de que los consumidores aprendan y se empoderen antes de introducir controles o mecanismos que restrinjan su capacidad de elección. En este aspecto, su postura sobre la intervención del Estado es mucho más cauta. 
Gráfica 1

Asimetría de información, riesgo y daño potencial en el consumo de bienes y servicios

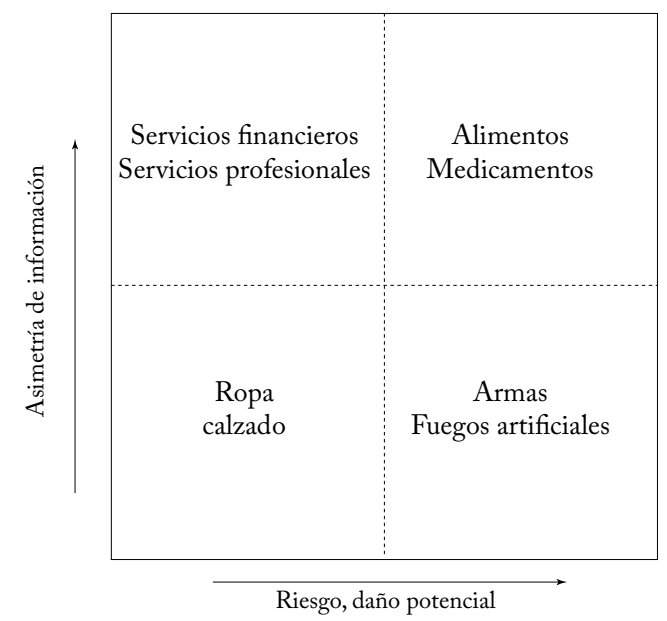

Fuente: elaboración propia.

de reiteradas experiencias de consumo resulta difícil para el consumidor apreciar las características relevantes del bien consumido. La existencia de asimetría de información, junto con el potencial riesgo asociado a la adopción de decisiones erróneas por parte del consumidor, define un espacio de situaciones en las cuales se justifica una mayor o menor intervención estatal mediante políticas de protección de los derechos del consumidor.

La gráfica 1 muestra de qué manera distintos grupos de bienes y/o servicios pueden justificar una mayor o menor intervención del Estado en función del grado de asimetría de información existente entre proveedor y consumidor, y su relación con el grado en que dicha actividad de consumo puede ocasionar un riesgo y un daño potencial para el consumidor.

Con relación a los riesgos y al daño potencial para el consumidor de consumir ciertos productos se acepta que la provisión de algunos bienes requiere un nivel mayor de vigilancia o control del Estado -p. ej., medicamentos, alimentos y armas-, y que los riesgos y el daño potencial justificando la existencia de mecanismos de regulación de la entrada a sus mercados. No ocurre lo mismo en el caso de otros bienes -p. ej., ropa y calzado- en los cuales los atributos relevantes para que un consumidor decida consumirlos son verificables rápida y fácilmente. Por otro lado, existen bienes y servicios cuyo consumo no tiene un alto daño potencial en los que puede haber una fuerte 
asimetría de información, como en ciertos servicios profesionales, médicos o financieros.

En cuanto al problema de la asimetría en la capacidad de negociación, un ejemplo para ilustrarlo se da en el caso de la contratación masiva de servicios. En estos casos, la necesidad de estandarización de los términos contractuales, de reducción de costos de transacción y aprovechamiento de economías de escala, ha llevado con frecuencia a la elaboración de contratos de adhesión. Más allá de que este proceso es connatural a la contratación masiva, pues sería imposible e ineficiente la existencia de una negociación bilateral para cada transacción en cada tipo de servicio, a menudo se aprecia el uso de cláusulas arbitrarias o contrarias a la prestación de un servicio idóneo. Por ello, en muchos países, las leyes de protección al consumidor establecen regulaciones que definen principios o lineamientos para la redacción de este tipo de cláusulas de contratación masiva. Asimismo, también es una práctica habitual en sectores regulados que las cláusulas generales de contratación deban contar con la aprobación previa de una agencia estatal.

Existen diversos estudios que documentan los efectos que tienen las políticas de protección al consumidor en países en desarrollo sobre el bienestar de los consumidores, pero en especial sobre su capacidad de elección. Witzke et al. (2005) demuestran que políticas de protección al consumidor que promueven la educación de los consumidores, junto con otras políticas públicas, han permitido la reducción de enfermedades gastrointestinales mortales. En otros sectores, como el financiero, también existen estudios que demuestran que la educación y el empoderamiento de los consumidores permiten integrar a sectores más pobres de la población al sistema financiero, dotándolos de una mayor capacidad de defensa de sus derechos (OECD et al., 2009).

Si bien en ciertos mercados, como el de alimentos y o medicamentos, se reconoce ampliamente que el Estado debe ejercer un control de la entrada dado el riesgo potencial que podría acarrear su consumo, en otras áreas ha existido debate respecto a si sería necesario un nivel de intervención aún mayor. Por ejemplo, el alcance de las políticas de consumidor en cuanto al consumo de tabaco (Prabhat et al., 2000) o alcohol (Cook y Moore, 2002), además de incluir el control de las condiciones de entrada, generalmente se ha extendido al control y regulación de la publicidad y el suministro de información al usuario.

Otra fuente importante de debate respecto al alcance de las políticas de protección al consumidor está vinculado con el supuesto de racionalidad detrás de las mismas. Por un lado, autores como Cvjetanovic (2014) o Engel (1995) cuestionan el uso del concepto 
de soberanía del consumidor para describir el comportamiento de un consumidor racional con una elevada capacidad de procesamiento de información, poniendo de relieve los desarrollos recientes en Economía del Comportamiento. En contraste, autores como Waldfogel (2005) o Saving (2006) han criticado el hecho de que con frecuencia se argumenta la falta de información o la racionalidad acotada de los consumidores para justificar políticas mediante las cuales el Estado termina adoptando decisiones en lugar del consumidor, sustituyéndolo y, a menudo, restringiendo su libertad de elección. Como parte de esta crítica se plantea que el Estado puede tener iguales o mayores restricciones informacionales que el consumidor a la hora de hacer la mejor elección. Waldfogel (2005) señala que la existencia de racionalidad acotada o falta de información no constituye un argumento suficiente que justifique la intervención del Estado, mostrando, a través de un experimento, que los consumidores otorgan un mayor valor a aquellas decisiones adoptadas por sí mismos. En la misma línea, Frey (2004) introduce el concepto de "utilidad procedimental", entendida como aquella que se desprende del bienestar que experimentan los consumidores como consecuencia de su nivel de participación y autonomía en las decisiones de consumo adoptadas.

Con frecuencia las intervenciones del Estado en materia de consumo han sido calificadas como 'paternalistas' en la medida que pueden interferir en las libertades de elección del consumidor (Smith y Sywicki, 2014). Al respecto algunos autores como Thomas y Buckmaster (2010), entre otros, han planteado que dicho concepto no puede generalizarse a cualquier intervención del Estado en materia de consumo, sino solo en la medida que esta no se base en la existencia de una falla de mercado, no esté basada en un riguroso análisis costobeneficio y cuyo proceso de decisión no goce de legitimidad. Por otro lado, desde la perspectiva de la soberanía del consumidor, intervenciones de distinto tipo que eficazmente prevengan o corrijan el engaño, el fraude u otros fenómenos que abordan las leyes de protección al consumidor serán deseables en la medida que incrementen la libertad de los consumidores en sus facetas de proceso y oportunidad.

El debate respecto al rol del Estado en la protección al consumidor y el nivel de racionalidad de este último aún no está agotado. Las conclusiones a las que arribe este debate determinarán en qué medida la "libertad de los consumidores" puede entenderse como una "libertad para equivocarse" o "adoptar decisiones erradas"; o, por el contrario, si existe un espacio de intervención del Estado para garantizar un piso mínimo para la adopción de decisiones de consumo adecuadamente 
informadas y el fortalecimiento de las libertades de oportunidad y proceso del consumidor.

Más allá de la discusión en torno al supuesto de racionalidad detrás del comportamiento del consumidor, hoy es universalmente aceptada la idea de que es necesario que existan normas que protejan al consumidor frente a los problemas de asimetría de información y de negociación. Como se ha mencionado, en la mayoría de países con economías de mercado existen leyes que protegen los derechos de los consumidores frente a los problemas informacionales y de asimetría en la capacidad de negociación, arriba mencionados. La protección de estos derechos dependerá también del acceso efectivo de los ciudadanos y consumidores a la justicia, y de un adecuado marco de cumplimiento de la ley. De la existencia de esos derechos y de su protección eficaz dependerá que la disponibilidad de una pluralidad de opciones se traduzca en una efectiva libertad de elección y en una efectiva soberanía del consumidor.

\section{SÍNTESIS: POLÍTICAS PÚBLICAS PARA PAÍSES EN DESARROLLO}

Como menciona Rothenberg (1962, p. 26), la aproximación al concepto de soberanía del consumidor puede ser de carácter descriptivo o normativo. La primera se centra en determinar de qué manera la producción y la oferta son efectivamente determinadas por las preferencias de los consumidores. La segunda postula que la soberanía del consumidor es un objetivo ideal de política, en virtud del cual el desempeño de la economía es susceptible de ser evaluado. En el presente artículo se ha optado por este último enfoque, que coincide con la óptica original del artículo de Hutt.

En las secciones anteriores se planteó que para que el mercado permita ejercer una auténtica libertad de elección a los consumidores y para que los patrones de producción respondan a sus preferencias, es preciso atenderles un conjunto de necesidades básicas, garantizarles el acceso a una pluralidad de opciones cuando sea posible y asegurarles el acceso a la justicia de consumo.

Desde una perspectiva de políticas públicas, cualquier agenda que plantee como objetivo una efectiva primacía del consumidor, a partir de la defensa de libertades individuales, debe dar prioridad a las que apunten a satisfacer las necesidades básicas que potencien sus capacidades de desarrollo; en segundo lugar a las políticas regulatorias de la competencia; y, en tercer lugar, a la eficaz protección de los derechos del consumidor, con base en rigurosos análisis costo-beneficio. 
Es difícil que los enfoques basados exclusivamente en el laissez faire garanticen que los mercados por sí solos aseguren que la soberanía del consumidor sea una consecuencia natural del ejercicio de una auténtica la libertad de elección.

De una adecuada y oportuna combinación de instrumentos de política que permitan asegurar un piso mínimo de igualdad de oportunidades y que apunten a garantizar las condiciones reseñadas en este artículo para asegurar la libertad de elección de todos los consumidores (tanto de proceso como de oportunidad) dependerá que los países en desarrollo se acerquen al ideal propuesto por Hutt: tener mercados donde sean los auténticos soberanos.

\section{ANEXO}

Gráfica A1

IDH y acceso a servicios de saneamiento

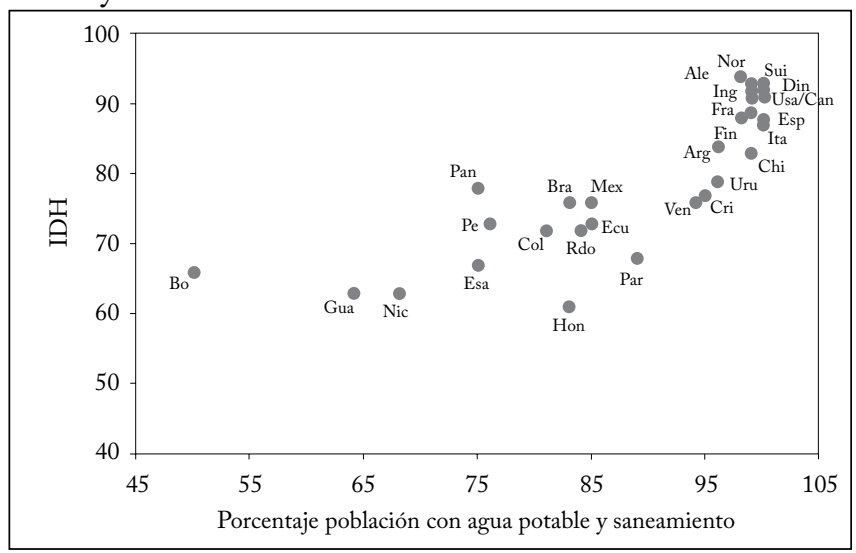

Fuente: PNUD y UNICEF, elaboración propia.

Gráfica A2

IDH y tasa de alfabetismo

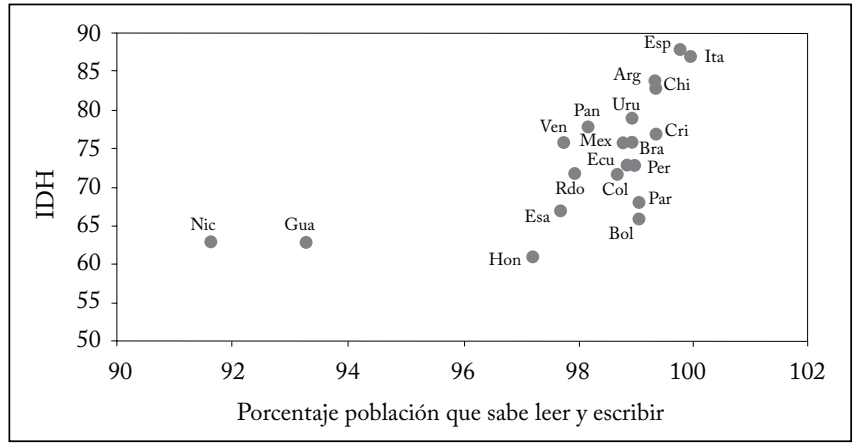

Fuente: PNUD y UNICEF, elaboración propia. 
Gráfica A3

IDH y acceso a energía eléctrica

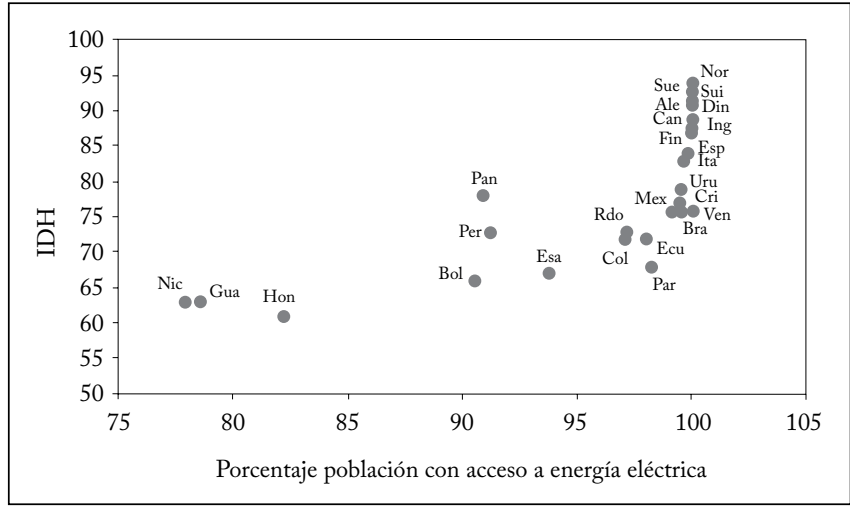

Fuente: PNUD y Banco Mundial, elaboración propia.

Gráfica A4

IDH e incidencia de TBC

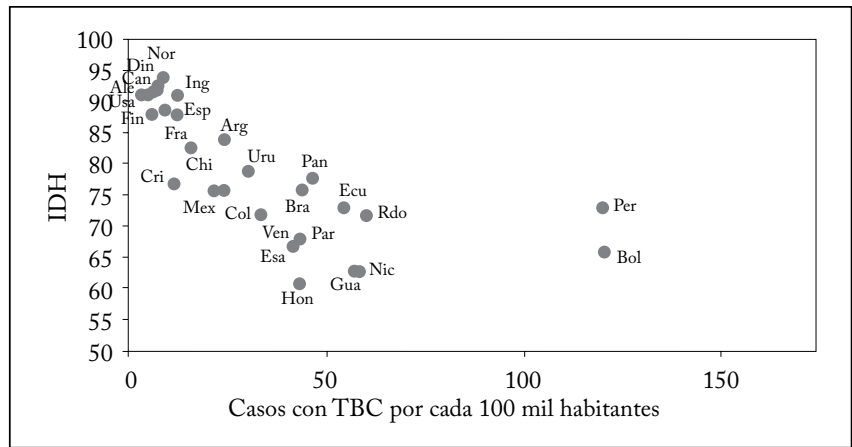

Fuente: PNUD y OMS, elaboración propia.

\section{REFERENCIAS BIBLIOGRÁFICAS}

Akerlof, G. A. (1970). The market for "lemons": Quality uncertainty and the market mechanism. The Quarterly Journal of Economics, 84(3), 488-500.

Cook, Ph. y Moore, M. (2002). The economics of alcohol abuse and alcohol-control policies. Health Affairs, 21(2), 120-133.

Cotter, Th. (2010). The essential facilities doctrine. Antitrust law and economics, vol. 4. K. Hylton (ed.), Encyclopedia of Law and Economics (pp. 157-182). Cheltenham: Edward Elgar.

Cseres, J. (2005). Competition policy and consumer protection. Ámsterdam: Kluwer.

Darby M. y Karni, E. (1973). Free competition and the optimal amount of fraud. Journal of Law and Economics, 16(1), 67. 
Engel, E. (1995). Consumer protection policy and rational behavior. Revista de Análisis Económico, 10(2), 183-202.

Feres, J. C. y Mancero, J. (2001). El método de las necesidades básicas insatisfechas y su aplicación en América Latina. Santiago de Chile: Cepal.

Filho, S. (2015). Monopolies and underdevelopment: From colonial past to global reality. Series New Horizons in Competition Law and Economics. Londres: Edward Elgar Publishing.

Frey, B., Benz, M. y Stuzer, A. (2004). Introducing procedural utility: Not only what, but also what matters. Journal of Institutional and Theoretical Economics, 160, 377-401.

Godfrey, N. (2008). Why is competition important for growth and poverty reduction? Conference Document, OECD Global Forum on International Investment, [http://www.oecd.org/investment/globalforum/40315399.pdf].

Gunning, P. (2009). Rothbard on consumer sovereignly and his implicit rejection of Mises's economics. Smithfield: Bryant University, [http://www. sdaeonline.org/wp-content/uploads/doc/Gunning_Rothbard_on_Mises.pdf].

Hayek, F. (1944). The road to serfdom. Nueva York: Routledge Press.

Hayek, F. (1960). The Constitution of liberty. Chicago: The University of Chicago Press.

Hutt, W. (1940a). The economist and the public. A study of competition and opinion. Londres: Transaction Publishers.

Hutt, W. (1940b). The concept of consumers' sovereignty. The Economic Journal, 50(197), 66-77.

Hutt, W. (1943). Plan for reconstruction. Londres: Kegan Paul \& Co.

Islam, S. y Clarke, M. (2001). The relationship between well-being, utility and capacities: A new approach to social welfare measurement based on Maslow's hierarchy of needs. Melbourne: Victoria University, [http://ged.u-bordeaux4.fr/SAMATTHEW.pdf].

Kenrick, D., Griskevicius V., Neuberg S. et al. (2010). Renovating the pyramid of needs: Contemporary extensions built upon ancient foundations. Perspectives on Psychological Science, 5(3), 292-314.

Maslow, A. (1943). A theory of human motivation. Psychological Review, 50, 370-396.

Nelson, Ph. (1970). Information and consumer behavior. Journal of Political Economy, 78(2), 311-329.

Nelson, Ph. (1974). Advertising as information. Journal of Political Economy, 82(4), 729-754.

Nozick, R. (1974). Anarquí, Estado y utopía. Madrid: Fondo de Cultura Económica.

OECD et al. (2009). Financial literacy in developing countries: Promoting access to finance by empowering consumers, [https://www. globalbrigades.org/media/Financial_Literacy.pdf].

Persky, J. (1993). Retrospective: Consumer sovereignty. Journal of Economic Perspectives, 7(1), 183-191.

Posner, R. (1974). The social cost of monopoly. NBER working paper 55. Chicago: The University of Chicago Press. 
Prabhat, J., Musgrove, Ph., Chaloupka, F. et al. (2000). The economic rationale for intervention in the tobacco market. En P. Jha y F. Chaloupka (eds.), Tobacco control in developing countries. Oxford: Oxford University Press.

Rawls, J. (1971). Una teoría de la justicia. Cambridge, Mass.: Harvard University Press.

Rawls, J. (1993). El liberalismo politico. Nueva York: Columbia University Press.

Roemer, J. y Tranoy, A. (2013). Equality of oportunity. En A. Atkinson y F. Bourguignon (eds.), Handbook of income distribution, vol 2. Amsterdam: North Holland

Rothenberg, J. (1962). Consumers' sovereignty and the hospitality of freedom of choice. The American Economic Review, 52(2), 269-283.

Saving, J. (2006). Consumer sovereignty in the modern global era. Journal of Private Enterprise, 22(1), 107-119.

Sen, A. (1993). Markets and freedoms: Achievements and limitations of the market mechanism in promoting individual freedoms. Oxford Economic Papers, 45(4), 519-541.

Sen, A. (2005). Human rights and capabilities. Journal of Human Development, 6(2),151-166.

Smith, A. (1776). An inquiry about the nature and causes of the wealth of nations, [http://www.ibiblio.org/ml/libri/s/SmithA_WealthNations_p. pdf].

Smith, A. y Todd, Z. (2014). Behavior, paternalism, and policy, evaluating consumer financial protection. Journal of Law and Liberty, Working paper, 14-06, March.

Thomas, M. y Buckmaster, L. (2010). Paternalism in social policy when is it justifiable? Research paper 8, Social Policy Section, Parliament of Australia.

Von Mises, L. (1949). Human action. Auburn: Ludwig von Mises Institute, [https://mises.org/sites/default/files/Human\%20Action_3.pdf].

Witzke von, H., Kirschke, D., Lotze-C., H. et al. (2005). The economics of consumer protection in developing countries' food economy: The case of diarrhea prevention and treatment in Rwanda. Agrarwirtschaft, 54, 314-319.

Wahba, M. y Bridwell, L. (1976). Maslow reconsidered: A review of research on the need hierarchy theory. Organizational Behavior and Human Performance, 15, 212-240.

Waldfogel, J. (2005). Does consumer irrationality trump consumer sovereignty? The Review of Economics and Statistics, 87(4), 691-696.

Wandel, J. (2012). Competition and antitrust policy: An Austrian economics perspective. En G. Leismuller y E. J. Schimpf (eds.), Economics of competition. Nueva York: Nova Science Publishers.

Williamson, O. (1968). Economies as an antitrust defense: The welfare trade-offs. American Economic Review, 58(1), 18-36.

Yamey, B. (1988). Professor Hutt on monopoly policy (1934-1943). Mangerial and Decision Economics, 9(5), 27-31. 